\title{
TOPOLOGICAL ENTROPY
}

BY

\section{R. L. ADLER, A. G. KONHEIM AND M. H. McANDREW}

Introduction. The purpose of this work is to introduce the notion of entropy as an invariant for continuous mappings.

1. Definitions and general properties. Let $X$ be a compact topological space.

Definition 1 . For any open cover $\mathfrak{A}$ of $X$ let $N(\mathfrak{A})$ denote the number of sets in a subcover of minimal cardinality. A subcover of a cover is minimal if no other subcover contains fewer members. Since $X$ is compact and $\mathfrak{U}$ is an open cover, there always exists a finite subcover. To conform with prior work in ergodic theory we call $H(\mathfrak{U})=\log N(\mathfrak{U})$ the entropy of $\mathfrak{A}$.

Definition 2. For any two covers $\mathfrak{A}, \mathfrak{B}, \mathfrak{A} \vee \mathfrak{B} \equiv\{A \cap B \mid A \in \mathfrak{A}, B \in \mathfrak{B}\}$ defines their join.

Definition 3. A cover $\mathfrak{B}$ is said to be a refinement of a cover $\mathfrak{A}, \mathfrak{A} \prec \mathfrak{B}$, if every member of $\mathfrak{B}$ is a subset of some member of $\mathfrak{A}$.

We have the following basic properties.

Property 00. The operation $v$ is commutative and associative.

Property 0 . The relation $\prec$ is a reflexive partial ordering $\left({ }^{1}\right)$ on the family of open covers of $X$.

Property 1. $\mathfrak{A} \prec \mathfrak{A}^{\prime}, \mathfrak{B} \prec \mathfrak{B}^{\prime} \Rightarrow \mathfrak{A} \vee \mathfrak{B} \prec \mathfrak{A}^{\prime} \vee \mathfrak{B}^{\prime}$.

Proof. Consider $A^{\prime} \cap B^{\prime} \in \mathfrak{A}^{\prime} \vee \mathfrak{B}^{\prime}$ where $A^{\prime} \in \mathfrak{A}^{\prime}$ and $B^{\prime} \in \mathfrak{B}^{\prime}$. By hypothesis there exists $A \in \mathfrak{U}$ and $B \in \mathfrak{B}$ such that $A^{\prime} \subseteq A, B^{\prime} \subseteq B$. Thus $A^{\prime} \cap B^{\prime} \subseteq A \cap B$ where $A \cap B \in \mathfrak{A}_{\vee} \mathfrak{B}$.

REMARK. With the proper substitutions of $\mathscr{U}, \mathfrak{B}$ and the cover $\{X\}$ in the statement above we obtain $\mathfrak{A} \prec \mathfrak{A} \vee \mathfrak{B}$ and $\mathfrak{B} \prec \mathfrak{A} \vee \mathfrak{B}$ which reveals that the family of open covers is a directed set with respect to the relation $\prec$.

Property 2. $\mathscr{U} \prec \mathfrak{B} \Rightarrow N(\mathfrak{A}) \leqq N(\mathfrak{B}), H(\mathfrak{A}) \leqq H(\mathfrak{B})$.

Proof. Let $\left\{B_{1}, \cdots, B_{N(\mathfrak{B})}\right\}$ be a minimal subcover of $\mathfrak{B}$. Since $\mathfrak{U}<\mathfrak{B}$. there exists a subcover $\left\{A_{1}, \cdots, A_{N(\mathfrak{B})}\right\}$ of $\mathfrak{A}$. Therefore $N(\mathfrak{A}) \leqq N(\mathfrak{B})$ and also $H(\mathfrak{A}) \leqq H(\mathfrak{B})$.

Property 3. $\mathfrak{A} \prec \mathfrak{B} \Rightarrow N(\mathscr{A} \vee \mathfrak{B})=N(\mathfrak{B}), H(\mathfrak{A} \vee \mathfrak{B})=H(\mathfrak{B})$.

Proof. It follows from Property 1 that $\mathscr{B} \prec \mathscr{A} \vee \mathfrak{B}$ so that $N(\mathfrak{B}) \leqq$ $N(\mathfrak{A} \vee \mathfrak{B})$. On the other hand $\mathfrak{B}>\mathscr{A} \vee \mathfrak{B}$ which is a consequence of the hypothesis. Thus $N(\mathscr{A} \vee \mathfrak{B}) \leqq N(\mathfrak{B})$.

Received by the editors August 21, 1963.

( $\left.{ }^{1}\right)$ For terminology see J. L. Kelley, General topology, Van Nostrand, New York, 1955. 
Property 4. $N(\mathscr{A} \vee \mathfrak{B}) \leqq N(\mathfrak{A}) \cdot N(\mathfrak{B})$ and $H(\mathscr{A} \vee \mathfrak{B}) \leqq H(\mathfrak{A})+H(\mathfrak{B})$.

Proof. Let $\left\{A_{1}, \cdots, A_{n(\mathbb{A})}\right\}$ be a minimal subcover of $\mathfrak{A}$ and $\left\{B_{1}, \cdots, B_{N(\mathfrak{B})}\right\}$ be a minimal subcover of $\mathfrak{B}$. Then $\left\{A_{i} \cap B_{j} \mid i=1, \ldots, N(\mathfrak{A}), j=1\right.$, $\ldots, N(\mathfrak{B})\}$ is a subcover of $\mathscr{A} \vee \mathfrak{B}$. Consequently $N(\mathfrak{A} \vee \mathfrak{B}) \leqq N(\mathfrak{A})$ $N(\mathfrak{B})$.

Let $\varphi$ be a continuous mapping of $X$ into itself.

If $\mathfrak{A}$ is an open cover of $X$ then from continuity, the family of $\varphi^{-1} \mathfrak{A}$ $=\left\{\varphi^{-1} A \mid A \in \mathscr{U}\right\}$ is again an open cover.

Property 5. $\mathscr{A} \prec \mathfrak{B} \Rightarrow \varphi^{-1} \mathfrak{A} \prec \varphi^{-1} \mathfrak{B}$.

PROPERTY 6. $\varphi^{-1}(\mathscr{A} \vee \mathfrak{B})=\varphi^{-1} \mathscr{U} \vee \varphi^{-1} \mathfrak{B}$.

Property 7. $N(\mathfrak{R}) \geqq N\left(\varphi^{-1} \mathfrak{A}\right)$.

Proof. Let $\left\{A_{1}, \cdots, A_{N(\mathscr{A})}\right\}$ be a minimal subcover of $\mathscr{A}$. Since $\left\{\varphi^{-1} A_{1}\right.$, $\left.\cdots, \varphi^{-1} A_{N(\mathfrak{A}}\right\}$ is a cover, possibly not minimal, we have $N\left(\varphi^{-1} \mathfrak{A}\right) \leqq N(\mathfrak{A})$.

REMARK. When $\varphi$ is onto then $N(\mathfrak{Q})=N\left(\varphi^{-1} \mathfrak{Q}\right)$.

PROPERTY 8.

$$
\lim _{n \rightarrow \infty} H\left({ }_{k=0}^{n-1} \varphi^{-k} \mathfrak{A}\right) / n=\lim _{n \rightarrow \infty} H\left(\mathfrak{A} \vee \varphi^{-1} \mathfrak{A} \vee \cdots \vee \varphi^{-n+1} \mathfrak{A}\right) / n
$$

exists and is finite.

Proof.

$H\left(\mathscr{A} \vee \cdots \vee \varphi^{-m-n+1} \mathfrak{A}\right)=H\left(\mathscr{A} \vee \cdots \vee \varphi^{-m+1} \mathfrak{A} \vee \varphi^{-m}\left(\mathscr{A} \vee \cdots \vee \varphi^{-n+1} \mathfrak{A}\right)\right)$

$$
\begin{aligned}
& \leqq H\left(\mathscr{A} \vee \cdots \vee \varphi^{-m+1} \mathfrak{A}\right)+H\left(\varphi^{-m}\left(\mathscr{A} \vee \cdots \vee \varphi^{-n+1} \mathfrak{A}\right)\right) \\
& \leqq H\left(\mathscr{A} \vee \cdots \vee \varphi^{-m+1} \mathfrak{A}\right)+H\left(\mathscr{A} \vee \cdots \vee \varphi^{-n+1} \mathfrak{A}\right) .
\end{aligned}
$$

The first equality follows from Property 6; the next inequality from Property 4; and the final inequality from Property 7.

Letting $H_{n}=H\left(\mathscr{A} \vee \cdots \vee \varphi^{-n+1} \mathfrak{Q}\right)$ we have $H_{m+n} \leqq H_{m}+H_{n}$ and $H_{n} \geqq 0$ for all positive integers $m, n$. It is a standard exercise in analysis to prove that $\lim _{n \rightarrow \infty} H_{n} / n$ exists and is finite.

Definition. The entropy $h(\varphi, \mathfrak{A})$ of a mapping $\varphi$ with respect to a cover $\mathfrak{A}$ is defined as $\lim _{n \rightarrow \infty} H\left(\mathscr{A} \vee \varphi^{-1} \mathfrak{A} \vee \cdots \vee \varphi^{-n+1} \mathfrak{A}\right) / n$.

Property 9. $h(\varphi, \mathfrak{A}) \leqq H(\mathfrak{A})$.

Proof. This follows from Properties 4 and 7.

Property 10. $\mathscr{A} \prec \mathfrak{B} \Rightarrow h(\varphi, \mathfrak{A}) \leqq h(\varphi, \mathfrak{B})$.

Proof. This follows from Properties 1,2 and 5.

Property 11. If $\varphi$ is a homeomorphism then $h(\varphi, \mathfrak{A})=h\left(\varphi^{-1}, \mathfrak{A}\right)$.

Proof.

$$
\begin{aligned}
H\left(\mathfrak{A} \vee \cdots \vee \varphi^{-n+1} \mathfrak{A}\right) & =H\left(\varphi^{n-1}\left(\mathscr{A} \vee \cdots \vee \varphi^{-n+1} \mathfrak{A}\right)\right) \\
& =H\left(\mathscr{A} \vee \varphi \mathfrak{A} \vee \cdots \vee \varphi^{n-1} \mathfrak{A}\right) \\
& =H\left(\mathscr{A} \vee\left(\varphi^{-1}\right)^{-1} \mathfrak{A} \vee \cdots \vee\left(\varphi^{-1}\right)^{-n+1} \mathfrak{A}\right) .
\end{aligned}
$$

Definition. The entropy $h(\varphi)$ of a mapping $\varphi$ is defined as the $\sup h(\varphi, \mathfrak{A})$ where the supremum is taken over all open covers $\mathcal{A}$. (Considering $\{\mathrm{h}(\varphi, \hat{\mathcal{Q}}) \mid \mathfrak{A}\}$ 
as a net, $h(\varphi)=\lim _{\mathfrak{M}} h(\varphi, \mathfrak{A})$.)

Definition. A sequence $\left\{\mathscr{U}_{n} \mid n=1,2, \cdots\right\}$ of open covers is refining if

(1) $\mathfrak{A}_{n} \prec \mathscr{A}_{n+1}$.

(2) For every open cover $\mathfrak{B}$ there exists $\mathfrak{A}_{n}$ such that $\mathfrak{B} \prec \mathfrak{A}_{n}$.

A refining sequence of covers when it exists simplifies the computation of entropy as the next property reveals.

PROPERTY 12. If $\left\{\mathscr{A}_{n}\right\}$ is a refining sequence of covers

$$
h(\varphi)=\lim _{n \rightarrow \infty} h\left(\varphi, \mathfrak{A}_{n}\right) .
$$

Proof. This property follows from Property 10 above.

\section{General theorems.}

THEOREM 1. Entropy is an invariant in the sense that $h\left(\psi \varphi \psi^{-1}\right)=h(\varphi)$ where $\varphi$ is a continuous mapping of $X$ into itself and $\psi$ is a homeomorphism of $X$ onto some $X^{\prime}$.

Proof.

$$
\begin{aligned}
h\left(\psi \varphi \psi^{-1}, \psi \mathfrak{A}\right) & =\lim _{n \rightarrow \infty} H\left(\psi \mathfrak{A} \vee \psi \varphi^{-1} \psi^{-1} \psi \mathfrak{A} \vee \cdots \vee \psi \varphi^{-n+1} \psi^{-1} \psi \mathfrak{A}\right) / n \\
& =\lim _{n \rightarrow \infty} H\left(\mathfrak{A} \vee \varphi^{-1} \mathfrak{A} \vee \cdots \vee \varphi^{-n+1} \mathfrak{A}\right) / n \\
& =h(\varphi, \mathfrak{A}) .
\end{aligned}
$$

As $\mathscr{A}$ ranges over all open covers of $X, \psi \mathfrak{U}$ ranges over all open covers of $X^{\prime}$ since $\psi$ is a homeomorphism; hence $h\left(\psi \varphi \psi^{-1}\right)=h(\varphi)$.

THEOREM 2. $h\left(\varphi^{k}\right)=k h(\varphi)$ for $k$ a positive integer.

Proof.

$$
\begin{aligned}
& h\left(\varphi^{k}\right) \geqq h\left(\varphi^{k}, \mathcal{A} \vee \varphi^{-1} \mathscr{A} \vee \cdots \vee \varphi^{-k+1} \mathscr{A}\right) \\
& =k \lim _{n \rightarrow \infty} H\left(\mathscr{A} \vee \varphi^{-1} \mathscr{A} \vee \cdots \vee \varphi^{-k+1} \mathscr{A} \vee \varphi^{-k} \mathscr{A} \vee \cdots\right. \\
& \left.\vee \varphi^{-2 k+1} \mathscr{A} \vee \cdots \vee \varphi^{-(n-1) k} \mathscr{A} \vee \cdots \vee \varphi^{-n k+1} \mathscr{Q}\right) / n k \\
& =k h(\varphi, \mathfrak{A})
\end{aligned}
$$

for any open cover $\mathscr{A}$. Thus $h\left(\varphi^{k}\right) \geqq k h(\varphi)$. On the other hand, since

$$
\begin{aligned}
\mathscr{A} \vee\left(\varphi^{k}\right)^{-1} \mathscr{A} \vee \cdots \vee\left(\varphi^{k}\right)^{-n+1} \mathfrak{A} & \prec \mathscr{A} \vee \varphi^{-1} \mathscr{A} \vee \cdots \vee \varphi^{-n k+1} \mathfrak{A}, \\
h(\varphi, \mathfrak{A}) & =\lim _{n \rightarrow \infty} H\left(\mathscr{A} \vee \varphi^{-1} \mathfrak{A} \vee \cdots \vee \varphi^{-n k+1} \mathfrak{A}\right) / n k \\
& \geqq \lim _{n \rightarrow \infty} H\left(\mathscr{A} \vee\left(\varphi^{k}\right)^{-1} \mathfrak{A} \vee \cdots \vee\left(\varphi^{k}\right)^{-n+1} \mathfrak{A}\right) / n k \\
& =h\left(\varphi^{k}, \mathfrak{A}\right) / k,
\end{aligned}
$$

for any open cover $\mathfrak{A}$; thus $k h(\varphi) \geqq h\left(\varphi^{k}\right)$. 
CoROllaRY. If $\varphi$ is a homeomorphism then $h\left(\varphi^{k}\right)=|k| h(\varphi)$ for any integer $k$.

Theorem 3. Let $X$ and $Y$ be two compact topological spaces. Let $\varphi_{1}$ be a continuous mapping of $X$ into itself and $\varphi_{2}$ a continuous mapping of $Y$ into itself. Then

$$
h\left(\varphi_{1} \times \varphi_{2}\right)=h\left(\varphi_{1}\right)+h\left(\varphi_{2}\right)
$$

where $\varphi_{1} \times \varphi_{2}$ is the continuous mapping of $X \times Y$ into itself defined by $\varphi_{1}$ $\times \varphi_{2}:(x, y) \rightarrow\left(\varphi_{1} x, \varphi_{2} y\right)$.

Proof. Open covers of $X \times Y$ of the form $\mathfrak{A} \times \mathfrak{B} \equiv\{A \times B \mid A \in \mathfrak{A}$, $B \in \mathfrak{B}\}$ have the property that $N_{X \times Y}(\mathfrak{A} \times \mathfrak{B})=N_{X}(\mathfrak{A}) \cdot N_{Y}(\mathfrak{B})$ and $(\mathfrak{A} \times \mathfrak{B}) \vee\left(\mathfrak{A}^{\prime} \times \mathfrak{B}^{\prime}\right)=\left(\mathfrak{A} \vee \mathfrak{A}^{\prime}\right) \times\left(\mathfrak{B} \vee \mathfrak{B}^{\prime}\right)$ where $\mathfrak{A}, \mathfrak{A}^{\prime}$ are covers of $X$ and $\mathfrak{B}, \mathfrak{B}^{\prime}$ are covers of $Y$. Although a cover itself usually indicates which space it covers, subscripts on $N$ signifying the set being covered can be employed as above in order to reduce ambiguity. Consequently,

$$
h\left(\varphi_{1} \times \varphi_{2}, \mathfrak{A} \times \mathfrak{B}\right)=h\left(\varphi_{1}, \mathfrak{A}\right)+h\left(\varphi_{2}, \mathfrak{B}\right)
$$

which implies $h\left(\varphi_{1} \times \varphi_{2}\right) \geqq h\left(\varphi_{1}\right)+h\left(\varphi_{2}\right)$. To establish the reverse inequality we need only show that for an arbitrary cover $\mathfrak{C}$ of $X \times Y$ there exists a refinement of the form $\mathfrak{A} \times \mathfrak{B}$ where $\mathscr{U}$ is a cover of $X$ and $\mathfrak{B}$ is a cover of $Y$. Since every open subset of $X \times Y$ is a union of rectangles $A \times B, A$ open subset of $X, B$ open subset of $Y$, we can obtain a refinement of $\mathbb{E}$ consisting only of open rectangles and from this choose a minimal subcover (₹'; i.e., (F' $=\left\{A_{1}^{\prime} \times B_{1}^{\prime}, \cdots, A_{N\left(\mathbb{S}^{\prime}\right)}^{\prime} \times B_{N\left(\mathfrak{S}^{\prime}\right)}^{\prime}\right\}$ and $\mathfrak{E} \prec \mathfrak{S}^{\prime}$. Let $\mathscr{\mathcal { F }}^{\prime}=\left\{A_{1}^{\prime}, \cdots, A_{N\left(\mathfrak{S}^{\prime}\right)}^{\prime}\right\}$ and $\mathfrak{B}^{\prime}=\left\{B_{1}^{\prime}, \cdots, B_{N\left(\mathfrak{C}^{\prime}\right)}^{\prime}\right\}$. Let $A_{x}$ be the intersection of all sets from $\mathscr{U}^{\prime}$ which contain the element $x \in X$ and $B_{y}$ be the intersection of all sets from $\mathfrak{B}^{\prime}$ which contain the element $y \in Y$. These newly defined sets are of course open, and we can choose a finite number of points $x_{1}, \cdots, x_{m}$ in $X$ and $y_{1}$, $\cdots, y_{n}$ in $Y$ such that $\mathfrak{A}=\left\{A_{x_{1}}, \cdots, A_{x_{n}}\right\}$ and $\mathfrak{B}=\left\{B_{y_{1}}, \cdots, B_{y_{n}}\right\}$ are covers of $X$ and $Y$ respectively. Consider any set $A_{x_{i}} \times B_{y_{j}} \in \mathfrak{A} \times \mathfrak{B}$. Since $\mathfrak{C}^{\prime}$ is a cover of $X \times Y,\left(x_{i}, y_{j}\right) \in A_{k}^{\prime} \times B_{k}^{\prime}$ for some integer $k$ between 1 and $N\left(\mathfrak{C}^{\prime}\right)$. Because $x_{i} \in A_{k}^{\prime}$ and $y_{j} \in B_{k}^{\prime}$ it follows that $A_{x_{i}} \subseteq A_{k}^{\prime}$ and $B_{y_{j}} \subseteq B_{k}^{\prime}$; that is $A_{x_{i}} \times B_{y_{j}} \subseteq A_{k}^{\prime} \times B_{k}^{\prime}$ which implies the desired result $\mathbb{S} \prec \mathfrak{S}^{\prime} \prec \mathfrak{A} \times \mathfrak{B}$.

In the next theorem we apply the elementary lemma.

Lemma. Suppose $\left\{a_{n}\right\}$ and $\left\{b_{n}\right\}$ are two sequences of real numbers not less than 1 such that $\lim _{n \rightarrow \infty}\left(\log a_{n}\right) / n=a$ and $\lim _{n \rightarrow \infty}\left(\log b_{n}\right) / n=b$ exist. Then $\lim _{n \rightarrow \infty} \log \left(a_{n}+b_{n}\right) / n=\max \{a, b\}$.

Proof. For any $c>a, b$ there exists an integer $n_{0}$ such that log $a_{n}<n c$ and $\log b_{n}<n c$ whenever $n \geqq n_{0}$. Thus $\log \left(a_{n}+b_{n}\right)<n c+\log 2$ for $n \geqq n_{0}$. Consequently

$$
a, b \leqq \lim _{n \rightarrow \infty} \inf \left(\log \left(a_{n}+b_{n}\right)\right) / n \leqq \lim _{n \rightarrow \infty} \sup \left(\log \left(a_{n}+b_{n}\right)\right) / n<c .
$$


Therefore $\lim _{n \rightarrow \infty}\left(\log \left(a_{n}+b_{n}\right)\right) / n=\max \{a, b\}$.

Theorem 4. Let $X_{1}$ and $X_{2}$ be two closed subsets of $X$ such that $X=X_{1} \cup X_{2}$ and $\varphi X_{1} \subseteq X_{1}, \varphi X_{2} \subseteq X_{2}$ for a continuous mapping $\varphi$ of $X$ into itself. Then

$$
h(\varphi)=\max \left\{h\left(\varphi_{1}\right), h\left(\varphi_{2}\right)\right\},
$$

where $\varphi_{1}$ and $\varphi_{2}$ are the restrictions of $\varphi$ to $X_{1}$ and $X_{2}$ respectively.

Proof. Let $i=1$ or 2 . For any open cover $\mathscr{A}$ of $X$ the family $(\mathfrak{A})_{i}$ $=\left\{A \cap X_{i} \mid A \in \mathfrak{A}\right\}$ defines an open cover of $X_{i}$; open in the subspace topology of $X_{i}$. Employing subscripts on $N$ to indicate the space whose cover is being counted we have $N_{i}\left((\mathfrak{A})_{i}\right) \leqq N(\mathfrak{Q})$. For open covers $\mathscr{A}$ and $\mathfrak{B}$ of $X$ we also have $(\mathscr{A} \vee \mathfrak{B})_{i}=(\mathfrak{A})_{i} \vee(\mathfrak{B})_{i}$. Furthermore $\varphi_{i}^{-1}(\mathfrak{A})_{i}=\left(\varphi^{-1} \mathfrak{A}\right)_{i}$ because of the invariance of $X_{i}$. Let $\mathfrak{A}_{i}$ be an arbitrary open cover of $X_{i}$, open in the subspace topology of $X_{i}$. There exists an open cover $\mathscr{U}$ of $X$ such that $(\mathfrak{A})_{i}=\mathfrak{A}_{i}$ : namely, $\mathfrak{A}=\left\{A \cup\left(X-X_{i}\right) \mid A \in \mathfrak{A}_{i}\right\}$.

$$
N_{i}\left(\bigvee_{k=0}^{n-1} \varphi_{i}^{-k} \mathfrak{A}_{i}\right)=N_{i}\left(\bigvee_{k=0}^{n-1}\left(\varphi^{-k} \mathfrak{A}\right)_{i}\right)=N_{i}\left(\left(\bigvee_{k=0}^{n-1} \varphi^{-k} \mathfrak{A}\right)_{i}\right) \leqq N\left(\bigvee_{k=0}^{n-1} \varphi^{-k} \mathfrak{A}\right) .
$$

Thus $h\left(\varphi_{i}, \mathfrak{A}_{i}\right) \leqq h(\varphi, \mathfrak{A})$. Hence $h\left(\varphi_{i}\right) \leqq h(\varphi)$. On the other hand for any open cover $\mathscr{A}$ of $X$ we have

$$
N\left(\bigvee_{k=0}^{n-1} \varphi^{-k} \mathfrak{U}\right) \leqq N_{1}\left(\bigvee_{k=0}^{n-1}\left(\varphi^{-k} \mathfrak{A}\right)_{1}\right)+N_{2}\left(\bigvee_{k=0}^{n-1}\left(\varphi^{-k} \mathfrak{A}\right)_{2}\right)
$$

and as before

$$
N_{i}\left(\left(\bigvee_{k=0}^{n-1} \varphi^{-k} \mathfrak{A}\right)_{i}\right)=N_{i}\left(\bigvee_{k=0}^{n-1} \varphi_{i}^{-k}(\mathfrak{A})_{i}\right), \quad i=1,2 ;
$$

whereupon

$$
\log N\left(\bigvee_{k=0}^{n-1} \varphi^{-k} \mathfrak{U}\right) \leqq \log \left[N_{1}\left(\bigvee_{k=0}^{n-1} \varphi_{1}^{-k}(\mathfrak{A})_{1}\right)+N_{2}\left(\bigvee_{k=0}^{n-1} \varphi_{2}^{-k}(\mathfrak{A})_{2}\right)\right]
$$

Now applying the lemma

$$
h(\varphi, \mathfrak{A}) \leqq \max \left\{h\left(\varphi_{1},(\mathfrak{A})_{1}\right), h\left(\varphi_{2},(\mathfrak{A})_{2}\right)\right\}
$$

which yields upon taking suprema

$$
h(\varphi) \leqq \max \left\{h\left(\varphi_{1}\right), h\left(\varphi_{2}\right)\right\} .
$$

TheOREM 5. Let $\sim$ be an equivalence relation on a compact set $X$. Let $\varphi$ be a continuous mapping of $X$ into itself such that $\varphi x \sim \varphi y$ if $x \sim y$. If $\tilde{\varphi}$ is the mapping of $X / \sim$ into itself defined by $\tilde{\varphi}_{\pi}=\pi \varphi$ where $\pi$ is the projection of $X$ onto $X / \sim$ then

$$
h(\tilde{\varphi}) \leqq h(\varphi) .
$$


Proof. Let $\tilde{\mathfrak{A}}$ be an open cover of $X / \sim$. Then $\pi^{-1} \tilde{\mathscr{A}}$ is an open cover of $X$ and $N_{X}\left(\pi^{-1} \widetilde{\mathfrak{Q}}\right)=N_{X / \sim}(\widetilde{\mathfrak{Q}})$. Therefore $h\left(\varphi, \pi^{-1} \widetilde{\mathfrak{A}}\right)=h(\widetilde{\varphi}, \widetilde{\mathfrak{Q}})$ and hence

$$
h(\varphi)=\sup _{\mathscr{A}} h(\varphi, \mathfrak{U}) \geqq \sup _{\widetilde{\mathfrak{I}}} h\left(\varphi, \pi^{-1 \widetilde{\mathscr{I}}}\right)=\sup _{\widetilde{\mathfrak{A}}} h(\widetilde{\varphi}, \widetilde{\mathfrak{A}})=h(\widetilde{\varphi}) .
$$

3. Computation of entropy in metric spaces. Let $X$ be a compact metric space with metric $d$.

Definition. The diameter $d(\mathfrak{A})$ of a cover $\mathfrak{A}$ is defined by

$$
d(\mathfrak{A})=\sup _{A \in \mathscr{I}} d(A)
$$

where $d(A)$ is the diameter of the set $A$.

Lebesgue's Covering Lemma. For every open cover $\mathfrak{A}$ of a compact metric space $X$ there exists $\epsilon>0$ such that if $U$ is a set with $d(U)<\epsilon$ then $U$ is contained in one of the members of $\Re$. The supremum of all such numbers $\epsilon$ is called the Lebesgue number of $\mathfrak{A}$.

Rephrasing of Lebesgue's Covering Lemma. For open covers $\mathfrak{A}$ and $\mathfrak{B}$ of $X$, if $d(\mathfrak{B})<$ Lebesgue number of $\mathfrak{A}$ then $\mathfrak{A} \prec \mathfrak{B}$.

CoRollary. If $\left\{\mathfrak{A}_{n}\right\}$ is a sequence of open covers such that

$$
\begin{aligned}
& \mathfrak{A}_{n} \prec \mathfrak{I}_{n+1}, \\
& d\left(\mathfrak{R}_{n}\right) \rightarrow 0, \quad \text { as } n \rightarrow \infty,
\end{aligned}
$$

then $\left\{\Re_{n}\right\}$ is a refining sequence.

REMARK. This corollary assures the existence of refining sequences in metric spaces. For example, the sequence $\left\{\mathfrak{U}_{n}\right\}$, where $\mathfrak{U}_{n}$ is the set of all spheres of diameter less than $1 / n$, is refining. In addition from any sequence $\left\{\mathfrak{B}_{n}\right\}$ of covers satisfying condition (2) of the corollary we can construct $\left\{\mathfrak{A}_{n} \mid \mathscr{A}_{n}=\mathrm{V}_{k=0}^{n} \mathfrak{B}_{k}\right\}$ which satisfies both (1) and (2) and thus is refining.

Example 1. If $\varphi$ is an isometry of $X$ onto itself then $h(\varphi)=0$.

Proof. Let $\mathfrak{A}_{p}$ be the family of all open sets of diameter less than $1 / p$. Such a family enjoys the property that $\mathfrak{A}_{p} \vee \mathfrak{A}_{p}=\mathfrak{A}_{p}$. Since $\varphi$ is an isometry, $\varphi^{-1} \mathfrak{A}_{p}=\mathfrak{A}_{p}$. This implies $\mathfrak{A}_{p}=\mathfrak{A}_{p} \vee \varphi^{-1} \mathfrak{A}_{p} \vee \cdots \vee \varphi^{-n+1} \mathfrak{A}_{p}$. Therefore $h\left(\varphi, \mathfrak{A}_{p}\right)=0$. According to the previous corollary $\left\{\mathfrak{A}_{p}\right\}$ is a refining sequence so that we can conclude $h(\varphi)=0$.

EXAMPLE 1a. Let $(X, \varphi)$ be an equicontinuous compact dynamical system, then $h(\varphi)=0$.

Proof. The metric $d^{\prime}$ defined by $d^{\prime}(x, y)=\sup _{-\infty<n<\infty} d\left(\varphi^{n} x, \varphi^{n} y\right)$ is equivalent to $d$. With respect to this new metric, $\varphi$ is an isometry and the above statement applies.

EXAmpLE 1b. Let $X$ be a compact separable topological group and 
$\varphi: x \rightarrow a x b, a, b \in X$. Then $h(\varphi)=0$.

Proof. $X$ is metrizable, say with metric $d$. The rotation $\varphi$ is an isometry with respect to the metric $d^{\prime}$ defined by $d^{\prime}(x, y)=\sup _{u, v \in X} d(u x v, u y v)$ which is equivalent to $d$.

EXAmple 2. Let $X=\left\{\left(x_{1}, x_{2}\right) \mid x_{1}^{2}+x_{2}^{2}=1\right\}$ be the unit circle. If $\varphi$ is a homeomorphism of $X$ onto itself then $h(\varphi)=0$.

Proof. Let $\mathfrak{U}_{p}$ be a covering of $X$ by intervals on $X$ of arc length $1 / p$. The covering $\mathfrak{A}_{p} \vee \varphi^{-1} \mathfrak{A}_{p} \vee \cdots \vee \varphi^{-n+1} \mathfrak{A}_{p}$ is a covering of $X$ by intervals and $N\left(\mathscr{A}_{p} \vee \cdots \vee \varphi^{-n+1} \mathscr{A}_{p}\right) \leqq n N\left(\mathscr{A}_{p}\right)$. Thus $h\left(\varphi, \mathscr{A}_{p}\right)=0$; and since $\left\{\mathfrak{A}_{p} \mid p=1\right.$, $2, \cdots\}$ is refining, $h(\varphi)=0$.

EXAmple 3. Expressing the space of two-sided infinite sequences of zeros and ones by $X=\prod_{i=\infty}^{\infty} X_{i}$ where $X_{i}=\{0,1\}$ and endowing $X_{i}$ with the discrete topology the space $X$ is compact in the cartesian product topology by virtue of the Tychonoff theorem. Let $(x)_{i}$ denote the $i$ th component of the sequence $x \in X$. Then cartesian product topology on $X$ is the same as that determined by the metric $d$ where

$$
d(x, y)=\sum_{-\infty}^{\infty}\left|(x)_{i}-(y)_{i}\right| / 2^{|i|} .
$$

Consider the homeomorphism $\varphi$ of $X$ onto itself called the shift and defined by $(\varphi x)_{i}=(x)_{i+1}$. Let $\mathfrak{U}=\left\{\left\{x \mid(x)_{0}=0\right\},\left\{x \mid(x)_{0}=1\right\}\right\}$ and

$$
\mathfrak{A}_{p}=\bigvee_{k=-p}^{p} \varphi^{k} \mathfrak{U}, \quad p=0,1,2, \cdots
$$

Since $d\left(\mathfrak{A}_{p}\right) \rightarrow 0$, as $n \rightarrow \infty$, the sequence $\left\{\mathscr{A}_{p}\right\}$ is refining. Next

$$
\begin{aligned}
h(\varphi, \mathfrak{U}) \leqq h\left(\varphi, \mathfrak{A}_{p}\right) & =\lim _{n \rightarrow \infty} H\left(\bigvee_{k=0}^{n-1} \varphi^{-k} \mathfrak{A}_{p}\right) / n \\
& =\lim _{n \rightarrow \infty} H\left(\underset{k=-p}{\mathrm{P}} \varphi^{k} \mathfrak{A} \vee \underset{k=-p-1}{\bigvee} \varphi^{k-1} \mathfrak{U} \vee \cdots \vee \underset{k=-p-n+1}{\bigvee} \varphi^{k} \mathfrak{A}\right) / n .
\end{aligned}
$$

From property (3) it then follows that

$$
\begin{aligned}
h\left(\varphi, \mathfrak{A}_{p}\right) & =\lim _{n \rightarrow \infty} H\left(\underset{k=-p-n+1}{\stackrel{p}{\mathrm{~V}}} \varphi^{k} \mathfrak{A}\right) / n \leqq \lim _{n \rightarrow \infty} H\left(\underset{k=-p-n+1}{\stackrel{-p}{\mathrm{~V}}} \varphi^{k} \mathfrak{A}\right) / n \\
& \left.=\lim _{n \rightarrow \infty} H\left(\underset{k=-n+1}{\stackrel{0}{\mathrm{~V}}} \varphi^{k} \mathfrak{A}\right)\right) / n=h(\varphi, \mathfrak{U}) .
\end{aligned}
$$

Counting reveals that $N\left(\bigvee_{k=0}^{-n+1} \varphi^{-k} \mathfrak{U}\right)=2^{n}$ so that $h(\varphi, \mathfrak{A})=\log 2$. Thus $h\left(\varphi, \mathfrak{A}_{p}\right)=\log 2$ which by the refining property of the sequence $\left\{\mathfrak{A}_{p}\right\}$ implies that $h(\phi)=\log 2$.

REMARK. If $X_{i}=\{0,1, \cdots, N-1\}$ above, then $h(\varphi)=\log N$. Furthermore 
if $X_{i}$ above is some compact Hausdorff space containing an infinite number of points, then $h(\varphi)=\infty$.

Example 4 . Let $X$ be the two dimensional-torus, i.e., $X=E^{2} / \sim$ where $E^{2}$ is the Euclidean plane and $\sim$ is the equivalence relation which identifies two points in the plane if their corresponding coordinates differ by integers. A metric on $X$ can be defined in terms of the metric on $E^{2}$ by taking the distance between two points of $X$ to be the minimum distance between any representatives of these points in $E^{2}$. A continuous group automorphism $\varphi$ of $X$ has a representation $\varphi:(x, y) \rightarrow(a x+b y, c x+d y)$ (additions $\bmod 1)$ where $\left(\begin{array}{c}a b \\ c d\end{array}\right)$ is a unimodular matrix $A$, that is, a matrix of integers with determinant \pm 1 . Suppose $A$ has two linearly independent characteristic vectors $\alpha, \beta$, associated with characteristic values $\lambda, \mu$, where $|\lambda| \geqq 1$. Then

$$
h(\varphi)=\log |\lambda| \text {. }
$$

Proof. Consider a covering of $E^{2}$ by all open parellelograms with sides parallel to $\alpha$ and $\beta$ and having length $1 / p$. Each set is a representative of an equivalence class of sets under $\sim$. Let $\mathfrak{A}_{p}$ be an open covering of $X$ by these equivalence classes. If $A$ is one of the above parallelograms then $\varphi^{-n} A$ is equivalent to a parallelogram having sides of length $|\lambda|^{n} / p$ and $|\lambda|^{-n} / p$ which are again parallel to characteristic vectors. Considering one parallelogram equivalent to one of the sets of $\mathfrak{A}_{p}, p>1$, it can be seen that it takes $|\lambda|^{n-1}$ parallelograms to cover it which are equivalent to sets in $\varphi^{-n+1} \mathfrak{Q}_{p}$. Thus

$$
p^{2}|\lambda|^{n-1} \leqq N\left(\mathscr{A}_{p} \vee \varphi^{-1} \mathfrak{A}_{p} \vee \cdots \vee \varphi^{-n+1} \mathscr{A}_{p}\right) \leqq|\lambda|^{n-1} N\left(\mathscr{A}_{p}\right)
$$

from which follows $h\left(\varphi, \mathscr{A}_{p}\right)=\log |\lambda|$, for $p>1$. Since $\left\{\mathfrak{A}_{p} \mid p=1,2, \cdots\right\}$ is a refining sequence we have $h(\varphi)=\log |\lambda|$.

REMARK. If $X$ is an $n$-dimensional torus and $\phi$ a continuous automorphism of $X$ determined by an $n$ by $n$ unimodular matrix having real characteristic values $\lambda_{1}, \cdots, \lambda_{n}$ and $n$ linearly independent characteristic vectors, then a similar argument yields

$$
h(\varphi)=\sum_{\left|\lambda_{i}\right| \geqq 1} \log |\lambda| \text {. }
$$

A curiosity based on the techniques of this work is the following.

THEOREM. Let $X$ be a compact metric space with an infinite number of points. Let $\varphi$ be a continuous mapping of $X$ into itself. For any open cover $\mathfrak{A}$ there exists a number $\delta>0$ (depending on $\mathfrak{P}$ and $\phi$ ) such that

$$
d\left(\varphi^{-1} \mathfrak{A} \vee \varphi^{-2} \mathfrak{A} \vee \cdots \vee \varphi^{-n} \mathfrak{U}\right) \geqq \delta>0
$$

for all $n$.

Proof. Suppose $d\left(\varphi^{-1} \mathfrak{U} \vee \cdots \vee \varphi^{-n} \mathscr{U}\right) \rightarrow 0$, as $n \rightarrow \infty$. There exists an integer $N$ such that if $n \geqq N$ then $d\left(\varphi^{-1} \mathscr{U} \vee \cdots \vee \varphi^{-n} \mathfrak{A}\right)$ is less than the 
Lebesgue number of $\mathfrak{A}$. Therefore $\mathfrak{A} \prec \varphi^{-1} \mathfrak{A} \vee \cdots \vee \varphi^{-n} \mathfrak{A}, n \geqq N$. Thus $N\left(\mathscr{A} \vee \varphi^{-1} \mathscr{A} \vee \cdots \vee \varphi^{-n} \mathfrak{A}\right)=N\left(\varphi^{-1} \mathscr{A} \vee \cdots \vee \varphi^{-n} \mathfrak{A}\right)=N\left(\mathscr{A} \vee \cdots \vee \varphi^{-n+1} \mathfrak{A}\right)$. By induction $N\left(\mathfrak{A} \vee \varphi^{-1} \mathfrak{A} \vee \cdots \vee \varphi^{-n} \mathfrak{A}\right)=N\left(\mathfrak{A} \vee \cdots \vee \varphi^{-N} \mathfrak{A}\right)$ for $n \geqq N$; that is $N\left(\mathscr{A} \vee \cdots \vee \varphi^{-n} \mathfrak{A}\right)$ is bounded, say by the number $M$. Choose $M+1$ distinct points $x_{1}, \cdots, x_{M+1}$ and let $n$ be so large that $d\left(\mathfrak{A} \vee \cdots \vee \varphi^{-n} \mathfrak{A}\right)$ $<\min _{1 \leqq i<j \leqq M+1} d\left(x_{i}, x_{j}\right)$. This is a contradiction because to cover $x_{1}, \cdots, x_{M+1}$ with sets whose diameters are smaller than $\min _{1 \leqq i<j \leqq M+1} d\left(x_{i}, x_{j}\right)$ requires at least $M+1$ sets.

4. Background and unsolved problems. In ergodic theory the notion of entropy for measure-preserving transformations has been extensively studied by the Russian school. The measure-theoretic entropy is defined as follows $[4]$. Let $(X, \mathfrak{E}, \mu)$ be a measure space with $X$ a set of points, (5 a sigma-field of measurable subsets of $X$, and $\mu$ a countably additive measure on $\mathbb{E}$ with $\mu(X)=1$. Let $\mathfrak{U}=\left\{A_{1}, \cdots, A_{n}\right\}$ be a finite measurable partition of $X$, i.e., $X=\bigcup_{i=1}^{n} A_{i}, A_{i} \in \mathfrak{A}, \mu\left(A_{i} \cap A_{j}\right)=0, i \neq j$. The measuretheoretic entropy $H_{\mu}(\mathfrak{A})$ is defined by

$$
H_{\mu}(\mathfrak{A}) \equiv-\sum_{i=1}^{n} \mu\left(A_{i}\right) \log \mu\left(A_{i}\right) .
$$

Again $\mathfrak{A} \vee \mathfrak{B}$ denotes the common refinement of two measurable partitions $\mathfrak{A}$ and $\mathfrak{B}$ and we have $H_{\mu}(\mathfrak{A} \vee \mathfrak{B}) \leqq H_{\mu}(\mathfrak{A})+H_{\mu}(\mathfrak{B})$. If $\varphi$ is a measurepreserving transformation then $H_{\mu}\left(\varphi^{-1} \mathfrak{A}\right)=H_{\mu}(\mathfrak{A})$ and the limit $h_{\mu}(\varphi, \mathfrak{A})$ $=\lim _{n \rightarrow \infty} H_{\mu}\left(\mathfrak{A} \vee \cdots \vee \varphi^{-n+1} \mathfrak{A}\right) / n$ exists for every finite measurable partition I.

The number $H_{\mu}(\mathfrak{Q})$ perhaps appears somewhat mysterious. However, note that if $\mu\left(A_{i}\right)=1 / n, i=1, \cdots, n$, then $H_{\mu}(\mathfrak{A})=\log n$. The contents of this present work indicate that $H_{\mu}(\mathfrak{A})$ is merely a delicate method of counting the number of sets in a partition in such a manner that the measures of the sets are given their appropriate weight in the tally. The quantity $h_{\mu}(\varphi)=\sup h_{\mu}(\varphi, \mathfrak{A})$ where the supremum is taken over all finite measurable partitions $\mathfrak{A}$ is called the entropy of $\varphi$. This number is a spatial isomorphism invariant for measure-preserving transformations on $(X, \mathfrak{E}, \mu)$. The following theorem of J. G. Sinaĩ $|4|$ is used to compute entropies.

Theorem. If $\mathfrak{A}^{*}$ is a measurable partition such that $\mathbb{E}$ is the sigma-field generated by the family of sets $\bigcup_{n=\infty}^{\infty} \varphi^{n} \mathfrak{A}^{*}$, then

$$
h_{\mu}(\varphi)=h_{\mu}(\varphi, \mathfrak{2} *) .
$$

The material in this paper we patterned after another theorem of this sort due to Rohlin [3].

THEOREM. If $\left\{\mathfrak{A}_{n} \mid n=1,2, \ldots\right\}$ is a sequence of partitions such that $\mathfrak{A}_{n} \prec \mathfrak{A}_{n+1}$ and the sigma-field generated by $\bigcup_{n=1}^{\infty} \mathfrak{A}_{n}$ is $\mathbb{E}$, then $h(\varphi)$ $=\lim _{n \rightarrow \infty} h\left(\varphi, \mathfrak{A}_{n}\right)$. 
Let us consider some examples.

1. Let $X$ be compact separable Abelian group and $\mu$ Haar measure. Suppose $\varphi: x \rightarrow a x$ then $h_{\mu}(\varphi)=0[3]$.

2. Let $X$ be the $n$-dimensional torus and $\mu$ Haar measure. Let $\varphi$ be a continuous automorphism on $X$ whose associated unimodular matrix has $n$ real characteristic values $\lambda_{1}, \cdots, \lambda_{n}$ and $n$ linearly independent character vectors, then

$$
h_{\mu}(\varphi)=\sum_{\left|\lambda_{i}\right| \geqq 1} \log \left|\lambda_{i}\right| \quad\lfloor 4] .
$$

3. Let $(X, \mathfrak{F}, \mu)=\coprod \coprod_{i=-\infty}^{\infty}\left(X_{i}, \mathfrak{F}_{i}, \mu_{i}\right)$, where $X_{i}=\{0,1\}, \mathfrak{E}_{i}=\{\varphi,\{0\}$, $\left.\{1\}, X_{i}\right\}$ and $\mu_{i}(\{0\})=p, \mu_{i}(\{1\})=1-p, 0 \leqq p \leqq 1, i=0 \pm 1, \pm 2, \cdots$. Let $\varphi$ be the shift transformation on $(X, \mathbb{E}, \mu)$, i.e., $(\varphi x)_{i}=(x)_{i+1}$. Then $h(\varphi)=-p \log p-(1-p) \log (1-p)\lfloor 1]$. If $X_{i}$ is considered to be a compact topological group then $\mu_{i}$ is Haar measure only when $p=1 / 2$. In this case $X$ is also a compact topological group with the direct product measure $\mu$ being its Haar measure. The mapping $\varphi$ is a continuous automorphism on $X$ and $h(\varphi)=\log 2$ which coincides with the maximum of $-p \log p-(1-p)$ $\cdot \log (1-p)$ for $0<p<1$.

We adopt the convention that $h_{\mu}$ denotes measure-theoretic entropy with respect to a measure $\mu$ while $h$ denotes topological entropy. Examination of examples having both topological and measure-theoretic aspects leads to some conjectures.

Conjecture 1. Let $X$ be a compact topological space and $\mu$ a regular measure. If $\varphi$ is a homeomorphism on $X$ and also a measure-preserving transformation then $h_{\mu}(\varphi) \leqq h(\varphi)$.

Conjecture 2. Let $X$ be a compact metric space and $\varphi$ a homeomorphism on $X$. A result of Kryloff and Bogoliouboff [2] states that there exists regular measures $\mu$ with respect to which $\varphi$ is measure preserving. Then $h(\varphi)=\sup h_{\mu}(\varphi)$ where the supremum is taken over all such invariant regular measures. If this is true, is there something special about the measure or measures where this supremum is assumed?

Conjecture 3 . Let $X$ be a compact separable group and $\varphi$ a continuous automorphism on $X$. Then

$$
h(\varphi)=h_{\mu}(\varphi)
$$

where $\mu$ is Haar measure.

In other directions we conjecture

Conjecture 4. Suppose $\left\{\varphi_{t}\right\}$ is a one parameter flow on a compact space $X$. Then $h\left(\varphi_{t}\right)=|t| h\left(\varphi_{1}\right)$. This is true for $t$ rational. Is it true for all $t$ ?

Conjecture 5. Suppose $X$ and $Y$ are compact topological spaces and $\left\{\varphi_{x} \mid x \in X\right\}$ is a family of homeomorphisms on $Y$ such that $\varphi:(x, y)$ $\rightarrow\left(x, \varphi_{x} y\right)$ is a continuous mapping of $X \times Y$ onto itself. Is 


$$
h(\phi)=\max _{x \in X} h\left(\varphi_{x}\right) ?
$$

From Theorem 4 this can be verified when $X$ is a finite set.

5. Conclusion. The notion of entropy has an abstract formulation which we have not dealt with here. It can be tailored to fit mappings on other mathematical structures. For example, let $G$ be an Abelian group; for a finite subgroup $\mathfrak{A}$ of $G$ let $N(\mathfrak{A})$ equal the order of $\mathfrak{A}$; let $\mathfrak{A} \vee \mathfrak{B}$ be the group generated by two finite subgroups $\mathfrak{A}$ and $\mathfrak{B}$ of $G$; let $\mathfrak{A} \prec \mathfrak{B}$ mean $\mathfrak{A}$ is a subgroup of $\mathfrak{B}$; and finally let $\varphi$ be an endomorphism of $G$. The basic properties now hold and we can define the entropy $h(\varphi, \mathfrak{A})$ of $\varphi$ with respect to a finite subgroup $\mathfrak{A}$. The entropy $h(\varphi)$ of $\varphi$ is then $\sup h(\varphi, \mathfrak{A})$ where supremum is taken over all finite subgroups $\mathfrak{A}$ of $G$; and analogies to the general theorems can be established.

We are grateful to the referee for correcting the proof of Theorem 5 .

\section{REFERENCES}

1. P. R. Halmos, Entropy in ergodic theory, mimeographed notes, Univ. of Chicago, Chicago, Ill., 1959.

2. N. Kryloff and N. Bogoliouboff, La théorie générale de la mesure dans son application à l'étude des systèmes dynamiques de la mécanique non linéaire, Ann. of Math. (1937), 65-113.

3. V. A. Rohlin, Entropy of metric automorphism, Dokl. Akad. Nauk SSSR 124(1959), 980-983.

4. Ja. G. Sinai, On the concept of entropy of a dynamical system, Dokl. Akad. Nauk SSSR 124(1959), 768-771.

International Business Machines Corporation, YoRKTOWN HeIGHTS, New YORK 